\title{
Atypical Supernumerary Phantom Limb and Phantom Limb Pain in a Patient With Spinal Cord Injury: Case Report
}

\author{
Ja Young Choi, $\mathrm{MD}^{1}$, Hyo In Kim, MD ${ }^{1}$, Kil Chan Lee, $\mathrm{MD}^{2}$, Zee-A Han, MD ${ }^{3}$
}

\begin{abstract}
${ }^{1}$ Department of Physical Medicine and Rehabilitation, National Health Insurance Corporation Ilsan Hospital, Goyang;
${ }^{2}$ Department of Rehabilitation Medicine, Yonsei University College of Medicine, Seoul;

${ }^{3}$ Department of Spinal Cord Injury Rehabilitation, National Rehabilitation Center, Seoul, Korea
\end{abstract}

\begin{abstract}
Supernumerary phantom limb (SPL) resulting from spinal cord lesions are very rare, with only sporadic and brief descriptions in the literature. Furthermore, the reported cases of SPL typically occurred in neurologically incomplete spinal cord patients. Here, we report a rare case of SPL with phantom limb pain that occurred after traumatic spinal cord injury in a neurologically complete patient. After a traffic accident, a 43-year-old man suffered a complete spinal cord injury with a C6 neurologic level of injury. SPL and associated phantom limb pain occurred 6 days after trauma onset. The patient felt the presence of an additional pair of legs that originated at the hip joints and extended medially, at equal lengths to the paralyzed legs. The intensity of SPL and associated phantom limb pain subsequently decreased after visual-tactile stimulation treatment, in which the patient visually identified the paralyzed limbs and then gently tapped them with a wooden stick. This improvement continued over the 2 months of inpatient treatment at our hospital and the presence of the SPLs was reduced to $20 \%$ of the real paralyzed legs. This is the first comprehensive report on SPLs of the lower extremities after neurologically complete spinal cord injury.
\end{abstract}

Keywords Phantom limb pain, Spinal cord injury, Tetraplegia, Neuropathic pain

\section{INTRODUCTION}

Phantom limbs are persistent postural and motor experiences of the limb after physical loss and are usually

Received January 31, 2013; Accepted May 20, 2013

Corresponding author: Zee-A Han

Department of Spinal Cord Injury Rehabilitation, National Rehabilitation Center, 58 Samgaksan-ro, Gangbuk-gu, Seoul 142-884, Korea

Tel: +82-2-901-1802, Fax: +82-2-901-1591, E-mail: zeeahan@catholic.ac.kr

(c) This is an open-access article distributed under the terms of the Creative Commons Attribution Non-Commercial License (http://creativecommons. org/licenses/by-nc/3.0) which permits unrestricted noncommercial use, distribution, and reproduction in any medium, provided the original work is properly cited.

Copyright $\odot 2013$ by Korean Academy of Rehabilitation Medicine described after amputation of the extremities [1]. In numerous neurologic diseases including stroke, epilepsy, and spinal cord injury (SCI), a slightly altered form of the phantom limb with distinctive experiences of an additional limb can occur. This phenomenon is much less frequent than phantom limb sensations that occur after amputation or various lesions of the neuraxis, and is called supernumerary phantom limb (SPL). By definition, SPL refers to the awareness of an illusory extra limb in addition to the real regular limbs [1]. The symptom has been described in a variety of different neurologic disorders, with right hemispheric stroke being the most common.

SPL resulting from spinal cord lesions are extremely 
rare. The first description of supernumerary limbs in traumatic SCI was made in 1975 and involved a C5 SCI patient who had illusory awareness of an extra pair of legs [2]. However, the mechanisms underlying SPL are unknown, and although the clinical significance is also yet to be described, the subjective reality of the additional limbs cause considerable distress in the afflicted patients. Here, we report a rare case of SPL with phantom limb pain in a patient with SCI with no prior history of psychiatric disease. This is the first comprehensive report on a SPL of the lower extremities after complete SCI.

\section{CASE REPORT}

A 43-year-old man was admitted to our hospital for intensive rehabilitation 115 days after a traffic accident. Cervical computed tomography (CT) and magnetic resonance imaging (MRI) on the day of the injury had shown a C6 vertebral body fracture as well as a cervical cord signal change on T2-weighted MRI images (Fig. 1A). He had undergone a C5-6-7 lateral mass fusion on the day of the injury. Six days after injury, he was transferred to the rehabilitation department. A subsequent C5-6-7-T1 anterior and posterior fusion was additionally performed 35 days after injury due to spinal instability detected on follow-up cervical spine X-ray (Fig. 2). There was no concomitant brain injury on brain CT scans and all cognitive evaluations including cognitive score subsets of the
Functional Independence Measure showed intact cognition. Other than a past history of type 2 diabetes mellitus, no other significant medical history including psychiatric history existed. The patient also had no history of drug or alcohol misuse.

According to the International Standards for Neurological Classification of Spinal Cord Injury, the patient had a complete lesion (American Spinal Injury Association impairment scale A [ASIA A]) with a C6 neurologic level of injury. According to neurologic exams performed 6 days after injury, the initial upper extremity motor score was 27 and the lower extremity motor score was 0 . These scores improved to 31 and 0 , respectively, at 115 days after injury when he was transferred to our hospital. Total light touch scores were initially 21 for the right side and 21 for left side and improved to 28 and 29, respectively, at 115 days follow-up. Total pin prick scores were initially 23 for the right side and 24 for the left side, but improved to 27 and 27, respectively on follow-up. There was no interval change in the neurologic exam at 6 months followup. Electrodiagnostic studies performed 2 months after injury revealed complete myelopathy and bilateral symmetric peripheral sensorimotor polyneuropathy. Due to concurrent diabetes related peripheral polyneuropathy, sensory nerve conduction studies were done, which showed low amplitudes in bilateral ulnar, superficial peroneal, and sural nerves. However, conduction velocities of all examined nerves and bulbocavernosus reflex laten-
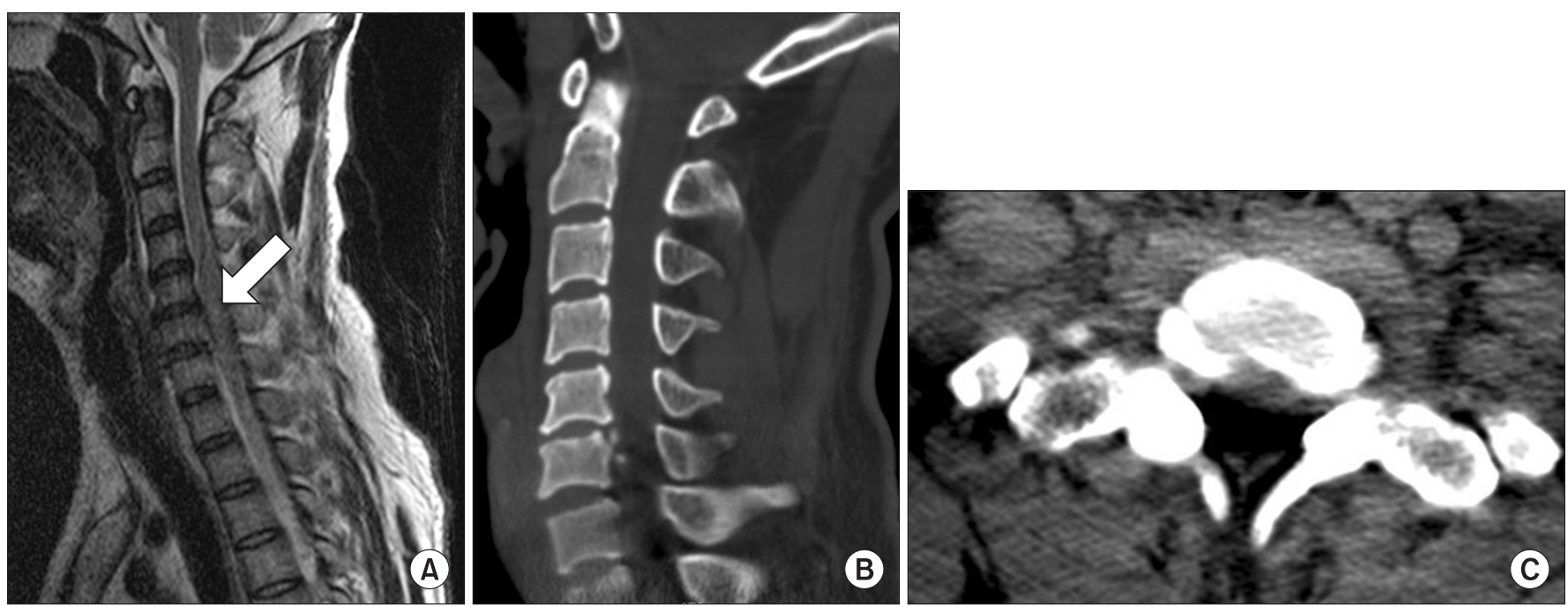

Fig. 1. Neuroimaging of the spinal cord on the day of injury. Magnetic resonance imaging scan T2-weighted sagittal view (A), showing cervical cord signal change (arrow). Computed tomography scan sagittal (B) and axial views (C), revealing C6 vertebral body fracture. 

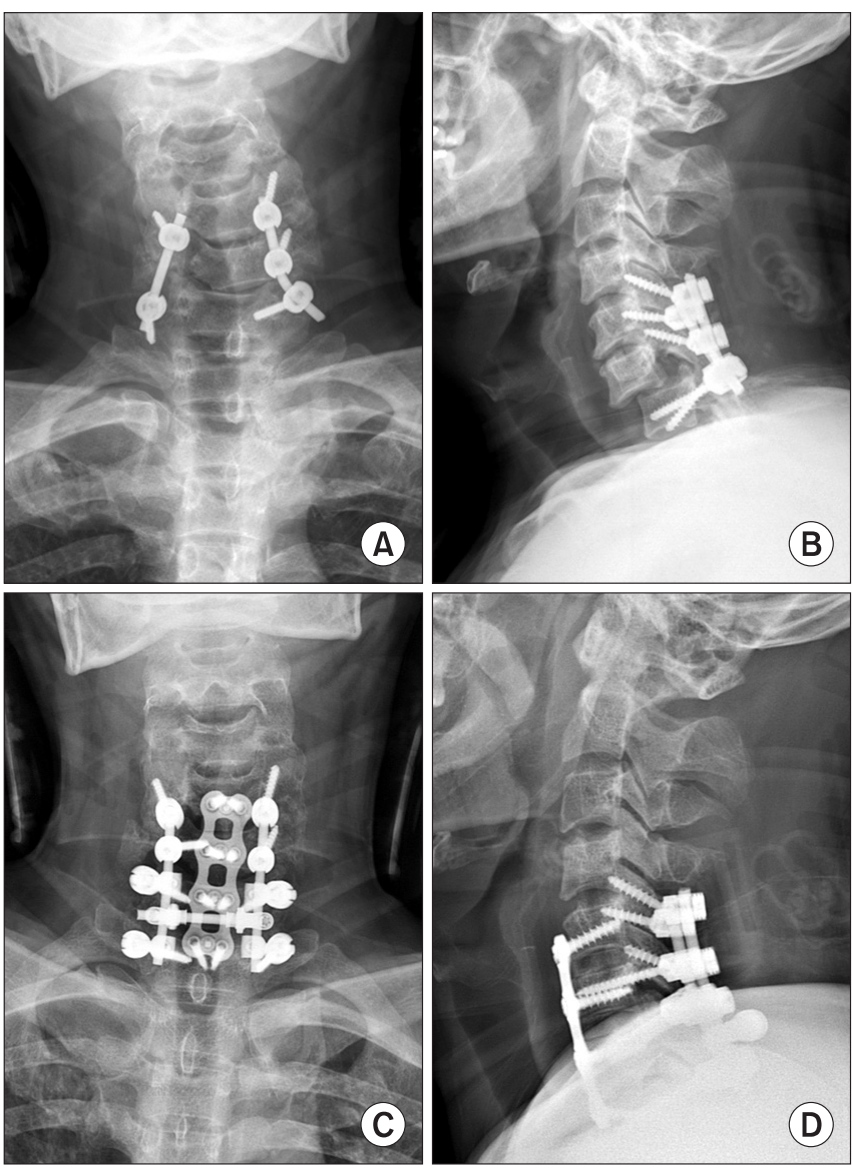

Fig. 2. Cervical spine X-ray anterior-posterior view (A) and lateral view (B) were taken after first operation, C56-7 lateral mass fusion, performed on the day of injury. Cervical spine X-ray anterior-posterior view (C) and lateral view (D) were taken after the second operation, C56-7-T1 anterior and posterior fusion, performed 35 days after injury.

cies were within normal range. Median somatosensory evoked potentials (SEP) stimulating the median nerves at the wrist bilaterally showed prolonged N20 latency in both sides. Tibial and pudendal SEPs were un-evoked with stimulations to the tibial nerves at the ankle and pudendal nerve at penile shaft.

SPL sensations appeared 6 days after trauma onset. The patient felt the presence of an additional pair of legs that originated at the hip joints and extended medially, at equal lengths to the paralyzed legs (Fig. 3). The patient described the presence of both knee and ankle joints in an extended position. SPL perceptions persisted throughout the day but its intensity fluctuated with more vividness during the afternoon and evening. Body posture also altered the intensity of SPL sensations with more

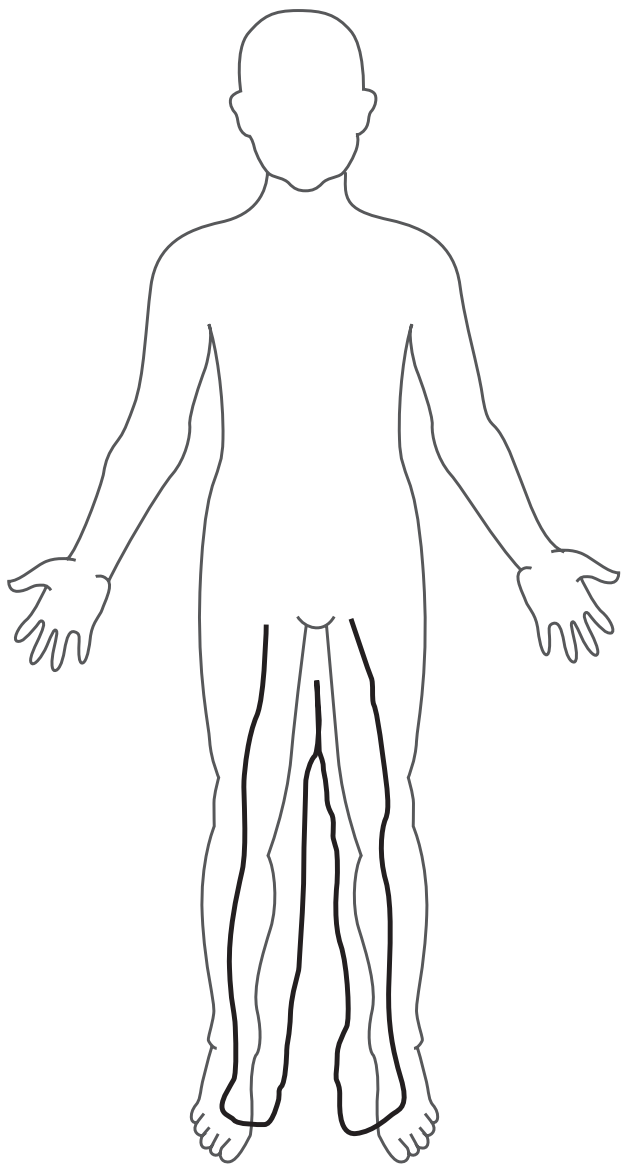

Fig. 3. Schematic representation of the phantom limb. Illusory lower limbs originate from the pelvic joints and extending at normal length medially to the patient's paralyzed legs.

prominence in the supine position and waning during sitting. The patient reported that he could feel but could not see the additional limbs and was aware that this feeling was an illusion. Visualization of his legs did not diminish or alter the intensity of SPL sensations. The supernumerary legs were also associated with severe lancinating pain that measured 8 points on the numeric rating scale. The patient also complained of severe neuropathic pain of the paralyzed legs that worsened when the SPLs were in the flexed position. However, he reported that he had no voluntary control over the phantom limbs and the movements of the SPLs were unintentional. Psychiatric evaluation showed no evidence of delusion, hallucination or any other specific psychiatric abnormality, and the patient showed good insight regarding his various clinical status related to his SCI. The intensity of the SPLs was equal to his paralyzed limbs. 
Combinations of oral gabapentin, pregabalin, baclofen, tramadol, and duloxetine improved the neuropathic pain in both the real and supernumerary limbs that measured 6 points on the numeric rating scale, but failed to affect changes in the intensity of the SPLs.

The patient underwent visual-tactile stimulation treatment where he would first visually identify his paralyzed limbs and then gently tap his legs with a wooden stick (Fig. 4). Although his legs were anesthetic to both pain and light touch, tactile stimulation evoked painful tingling sensations in his paralyzed legs. Thus, he was able to receive both visual and sensory feedback and reconfirm the existence of his paralyzed legs. Wooden stick stimulation also evoked painful tingling in the SPLs but visually he was able to recognize the absence of the SPLs and thus differentiate the sense as erroneous. Visualtactile stimulation was performed three times daily for 10-15 minutes per session with approximately one tap per second. The patient was then asked to rate the intensity of the SPLs on a 10-point numeric analog scale relative to the paralyzed real limb. The presence of the SPLs were felt at an equal intensity to the real limbs initially but improved to half the intensity of the real paralyzed limbs after 8 days of daily visual-tactile stimulation. This improvement continued over the 2 months of inpatient treatment at our hospital and the presence of the SPLs was reduced to $20 \%$ of the real paralyzed legs.

Nine months after the SCI, the patient reported persistence of the illusory limb sensations, but the sensations had improved to an extent where it no longer affected the patient's quality of life. Through a telephone interview

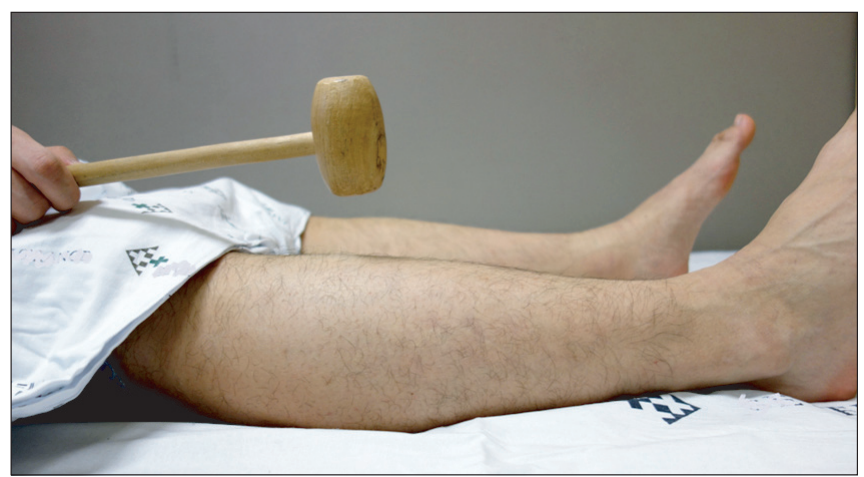

Fig. 4. Visual-tactile stimulation treatment. The patient would first visually identify his paralyzed limbs and then gently tap his legs with a wooden stick. conducted 13 months post-trauma, the patient reported a telescoping pattern of the SPL with total length shortening to levels half of the original. However, although shorter in length, the knee and ankle joints were still present and the limb continued to remain in the extended position. Although SPL sensation and subsequent total motor and sensory scores at 6 months follow-up improved, motor and sensory scores pertaining to the lower extremities as well as the ASIA A neurologically complete injury state remained unchanged. Additional lower limb SEP studies and imaging studies were not preformed due to patient refusal. However, considering the lack of change in both motor and sensory scores as well as ASIA grades, these studies did not appear to be clinically significant or essential.

\section{DISCUSSION}

Phantom limb phenomenon occurs in as many as $90 \%-100 \%$ of amputation cases [3]. However, such sensory alterations of deafferented limbs, which also occur in SCI patients, rarely manifest as a phantom limb. The SPL phenomenon is a subjective sensation of additional limbs that have occasionally been reported in central nervous system disorders [4]. Cortical damage or subcortical lesions have been associated with SPL, but SPL after spinal cord lesions have been extremely rare.

Supernumerary limbs in SCI was first mentioned in 1975 [2], and only sporadic and brief descriptions have been made in the literature since [5]. The latter review reported four cases with three of the cases occurring in cervical incomplete SCI patients [5].

Although phenomenologically similar, phantom sensations occurring after limb loss or after SCI and those felt after cerebral lesions may differ in several aspects. Pain is frequently associated with phantom limbs after amputation, but rarely accompanies SPLs [6]. Moreover, SPLs after brain damage may be associated with cognitive deficits, delusion or brief episodic seizures [7]. Although amputation was not the triggering event for SPL in our case, clinical manifestations were similar to amputation related phantom pain in that there was debilitating pain of the SPL in the absence of any consequences related to brain damage.

Head and Holmes [8] were the first to systematically study bodily perception or body schema and hypoth- 
esized that spatial perceptions of the body are achieved through central integration of incoming afferent information from the periphery and updated through successive changes in position. Thus, body schema is achieved through interplay between sensory feedback from the peripheral nervous system or periphery and image formulation originating from the non-dominant parietal lobe and other central brain structures [8].

SPLs and phantom limb sensations are thought to be a disruption of such normal bodily perceptions. SPLs are not infrequently reported after brain pathology and the main postulation is thought to be due to reorganization of the primary somatosensory cortex and subsequent deterioration of normal body schema [9].

However, the literature suggests a large spectrum of features of various types of SPLs differing by the degree of motor controllability (impossible, automatic, intentional movements), sensitivity to sensory feedback (visual, tactile, proprioceptive), and patients' mental framework (confused, delusional, indifferent) [9]. In this respect, it appears inappropriate to expect a single explanation, and mechanisms may involve both psychological and organic.

Other classic explanations, which may be more appropriate to our case, emphasize the dissociation between the previously established sensorimotor representations and the lesion-induced change in communication between the brain and the paralyzed limb [7]. In other words, these illusory phenomena might be provoked by loss of afferent information due to sensory impairment and failure to correctly integrate multimodal and motor information to generate a normal experience of selflocation [10]. Just as destruction of the sensory roots often leads to the phenomenological experience of a supernumerary limb, severe sensory and proprioceptive loss may erroneously re-program the innate body image into constructing an illusory limb. In amputees and SCI patients, the ability to command motor function of the limbs is either physically lost or neurologically disconnected. Hence, mismatch between central (cortical) movement and sensory feedback from the peripheral nervous system makes it difficult to distinguish between expected movements of the actual limbs with the unexpected movement of illusory limbs. Another relatively simple mechanism to explain SPL in SCI may be through subcortical brain reorganization following SCI.
Despite the many hypotheses, the underlying neural mechanisms of SPLs in spinal cord injury are not fully understood.

Phantom limb pain is a challenging condition and standardized treatment protocols for SPLs are not available. Clinicians have outlined the fact that phantom limb pain is difficult to treat in comparison to conventional pain. The critical clue for treatment is considering possible roles of the visual system in the elicitation of this perceptive mismatch. Some SPLs are reported as not seen and not influenced, either by visual feedback of the actual limbs or direct touch of the actual limb. But, in certain patients, the SPL or their associated features such as pain were eliminated through visual feedback and tactile stimulation of the real limb [6]. Distressing intensities of SPL sensation in our patient also improved through a regimen of visual-tactile stimulation. Although neuropathic pain of the paralyzed and SPLs improved with medication only stimulation treatment was effective in improving the intensity of the SPLs. Specific treatment protocols have not yet been developed. Therefore, visual-tactile stimulation treatment may be effective in improving the intensity of the SPLs in future patients. This treatment tool has the advantage of being convenient to apply and to carry out in both lower cervical SCI and paraplegia patients. However, further studies need to be done to reveal basic neurophysiological mechanisms of this interesting phenomenon and find effective measures to treat SPLs.

The patient reported here had the classic features of SPL. The phantom perception was vivid, continuous and not visualized, and the symptoms developed without any underlying cognitive or psychological dysfunction. Thus, an organic cause rather than a psychodynamic explanation was more appropriate in explaining the SPL in this patient.

Though there have been other case reports of supernumerary limbs in SCI patients, this case is prominent in many aspects. First, this case is the first to comprehensively report on SPL after complete SCI. Almost all reported case reports of SPL in SCI were associated with incomplete injury, except one single sentence mentioning of a case in 1975. Since the previous case was associated with incomplete SCI injury, the SPL sensation vanished with subsequent neurologic recovery. However, the patient in this case experienced SPLs in both lower extremities despite the neurological completeness of the SCI lesion. 
While the intensity did improve, the SPL persisted on subsequent follow-up. Second, SPL in this case appeared in bilateral lower extremities. Most of the reported cases of SPL in cervical SCI were present in the upper extremities including the most recent review article [5]. Third, a telescoping phenomenon, which is often experienced in amputees, was present in this case. This may implicate that the basic neurophysiological mechanism underlying phantom limbs of complete SCI patients may overlap those of amputees. Fourth, visual-tactile stimulation treatment, which is effective in amputee patients, was applied to our patient with relative success.

\section{CONFLICT OF INTEREST}

No potential conflict of interest relevant to this article was reported.

\section{REFERENCES}

1. Brugger P. Phantom limb to phantom body. In: Knoblich G, Thornton IM, Grosjean M, Shiffrar M, editors. Human body perception from the inside out. Oxford: Oxford University Press; 2005. p. 171-209.

2. Davis R. Pain and suffering following spinal cord injury. Clin Orthop Relat Res 1975;(112):76-80.
3. Fredericks J. Phantom limb and phantom limb pain. In: Fredericks J, editor. Clinical neuropsychology. Amsterdam: Elsevier; 1985. p. 373-93.

4. Halligan PW, Marshall JC, Wade DT. Three arms: a case study of supernumerary phantom limb after right hemisphere stroke. J Neurol Neurosurg Psychiatry 1993;56:159-66.

5. Curt A, Yengue CN, Hilti LM, Brugger P. Supernumerary phantom limbs in spinal cord injury. Spinal Cord 2011;49:588-95.

6. Grossi D, Di Cesare G, Tamburro RP. On the syndrome of the "spare limb": one case. Percept Mot Skills 2002;94:476-8.

7. Brugger P. Supernumerary phantoms: a comment on Grossi, et al.'s (2002) spare thoughts on spare limbs. Percept Mot Skills 2003;97:3-10.

8. Head H, Holmes G. Sensory disturbances from cerebral lesions. Brain 1911;34:102-254.

9. Khateb A, Simon SR, Dieguez S, Lazeyras F, MomjianMayor I, Blanke O, et al. Seeing the phantom: a functional magnetic resonance imaging study of a supernumerary phantom limb. Ann Neurol 2009;65:698-705.

10. Blanke O, Landis T, Spinelli L, Seeck M. Out-of-body experience and autoscopy of neurological origin. Brain 2004;127(Pt 2):243-58. 